\title{
EVALUATION OF $\beta$-CATENIN EXPRESSION IN DENTAL PULP FOLLOWING DIRECT PULP CAPPING IN DOG TEETH
}

\author{
Dalia A El-Baz*, Maha A El-Baz ${ }^{* *}$, Geraldine M Ahmed ${ }^{* * * *}$ and Alaa A El-Baz ${ }^{* * *}$
}

\begin{abstract}
Objective: The aim of the present study was to evaluate pulpal responses and $\beta$-catenin expression following direct pulp capping of mechanically exposed teeth with mineral trioxide aggregate (MTA), bioaggregate (BA) and stem cells (BM-MSCs) in dog teeth.

Methods: Six healthy male mongrel dogs, aged between 2.5 and 3.5 years of age, were used for the present experimental work. Permanent mandibular molars and premolars were selected as the most suitable teeth. Teeth were divided into three groups according to the capping materials. Occlusal pulp exposures were performed. The pulp exposures in each dog were randomly capped in pairs according to the manufacturer instructions with MTA( group I), Bioaggregate (group II) and Bone marrow-derived mesenchymal stem cells (group III), where MTA was used as the control and placed in a single pair of teeth. The cavities were restored with zinc oxide-eugenol and amalgam. The pulpal tissue responses to the tested materials were assessed postoperatively after sacrificing the dogs after 30 days. Real Time PCR for quantitative expression of $\beta$-catenin was used. One Way ANOVA was used to compare between the tested materials followed by Tukey's Post Hoc test for pairwise comparison.
\end{abstract}

Results: Group III showed the highest expression of $\beta$-catenin protein $(5.63 \pm 0.09)$ followed by group II $(3.42 \pm 0.17)$ followed by group I $(2.34 \pm 0.16)$ at $\mathrm{p} \leq 0.001$.

Conclusion: Dentinogenesis process was most active in group III (BM-MSCs) in comparison to both group I (MTA) and group II(BA) groups.

Keywords: Mineral Trioxide Aggregate, Bioaggregate, BM-MSCs, direct pulp capping

\section{INTRODUCTION}

Vital pulp therapy helps in increasing the expected life of the tooth by preserving a healthy pulp status. Once a tooth is affected by caries, some form of intervention is necessary to prevent further inflammation and ultimately necrosis. Pulp capping is indicated for pulp exposure after trauma or injury. This procedure offers good alternatives to endodontic therapy for teeth with immature

\footnotetext{
* Associate Professor, Oral Histology Department, Faculty of Oral and Dental Medicine, Cairo University

** Lecturer, Operative Department, Faculty of Oral and Dental Medicine, Cairo University

*** Associate Professor, Endodontic Department, Faculty of Oral and Dental Medicine, Cairo University
} 
or mature apices offering a more conservative approach by promoting the dentinogenic potential of the pulpal cells ${ }^{(1)}$.

Several factors affect the success of vital pulp therapy, most importantly is the status of the pulp tissue. The presence of adequate blood supply is required to maintain pulp vitality, the presence of a healthy periodontium and coronal seal to prevent bacterial microleakage ${ }^{(2-4)}$.

Many materials have been used for these procedures, including resin-modified glass ionomer cement, tricalcium phosphates, hydrophilic resins, and calcium hydroxide. The success of any pulp capping material has been measured by the thickness and morphology of the dentin bridge formed, intensity of pulpal inflammation, presence of odontoblasts cells, and biocompatibility ${ }^{(5)}$.

Historically calcium hydroxide has been considered the gold standard for pulp capping. However, previous research has shown that it is not ideally suited for this procedure due to the porosity of the dentin bridge produced, poor adherence to dentin and inability to provide a long-term seal. The porosity of the dentin bridge allows recolonization of bacteria leading to failure of pulp capping procedures ${ }^{(6)}$. Dammaschke et al. 2010, have reported that calcium hydroxide caused a layer of the pulp tissue necrosis when used as a pulp capping material $^{(7)}$.

Mineral trioxide aggregate (MTA) has been used as an alternative to calcium hydroxide as it contains tricalcium silicate, tricalcium aluminate, tricalcium oxide and silicate oxide ${ }^{(8)}$.

MTA has demonstrated significantly greater frequency of dentin bridge formation, thicker and less porous dentin, and less pulp inflammation compared with calcium hydroxide ${ }^{(9-11)}$. Recent research has shown that MTA, when placed in direct contact with the human dental pulp cells, differentiated them into odontoblast-like cells ${ }^{(12)}$. Compared with calcium hydroxide no layer of necrosis was seen using $\operatorname{MTA}^{(7)}$. MTA induced recruitment and proliferation of undifferentiated cells to form a dentin bridge while reducing inflammation ${ }^{(5)}$. Another study showed that MTA causes neutrophils recruitment to the site of injury, which is important in the process of inflammation ${ }^{(13)}$. Nair et al. 2008, also demonstrated decreased inflammation when MTA was used compared with calcium hydroxide ${ }^{(14)}$. On the molecular level, MTA induced the secretion of angiogenic factors, such as vascular endothelial growth factor, which plays a leading role in healing ${ }^{(15,16)}$.

Other studies reported that MTA induces cells to secrete IL-8 and IL-1b which takes a leading role in collagen synthesis, resulting in a more organized pulp response which assists in the healing process ${ }^{(17-19)}$.

Other studies in animal models have shown that MTA down-regulated inflammatory cytokines, such as interferon-gamma, and IL-1a and suppressed the proliferation of some microorganisms and inhibited the production of certain $\mathrm{TH} 1$ and $\mathrm{TH} 2$ cytokines $(20,21)$.

BioAggregate (BA) a white nanoparticle ceramic cement, consisting of tricalcium silicate, dicalcium silicate, calcium phosphate monobasic, amorphous silicone dioxide, and tantalum oxide as a radiopacifier have been used as an alternative to MTA ${ }^{(22)}$. BA showed similar cytotoxicity ${ }^{(23,24)}$, and biocompatibility levels to MTA ${ }^{(25)}$ by upregulating the gene expression of collagen 1, osteocalcin, and osteopontin in osteoblasts and differentiation of human periodontal ligament fibroblasts ${ }^{(26,27,28)}$.

Stem cells are non-specialized cells that divide continuously. They have the ability of self-renewal and capable of generating complex tissues and organs ${ }^{(29)}$. They are classified as either embryonic or postnatal ${ }^{(30)}$. The clinical applications of stem cells depend on their proliferation rate, differentiation potential, and accessibility. 
Currently, clinical endodontics include procedures that are based on the ability of stem cells to accomplish repairs such as direct pulp capping, apexogenesis, apexification, and even pulpal regeneration ${ }^{(31)}$.

$\beta$-catenin is a protein which plays fundamental roles in various biological processes such as tooth development. For instance, inactivation of $\beta$ -catenin in mesenchyme of developing tooth results in arrested tooth development at the bud stage. ${ }^{(32)}$.

The aim of the present study was to evaluate pulpal responses and $\beta$-catenin expression following direct pulp capping of mechanically exposed teeth with MTA, bioaggregate and Bone marrow-derived mesenchymal stem cells (BM-MSCs) in dog teeth.

\section{MATERIALS AND METHODS}

Six healthy male mongrel dogs, aged between 2.5 and 3.5 years of age, were used for the present experimental work. The experimental protocol was conducted according to the ethical guidelines for animal care in the Faculty of Oral and Dental Medicine, Cairo University. All animals were checked by the veterinarian to be clinically healthy. The animals were placed in labeled cages fed (supplied with regular hygienic diet and drinking water) and maintained along the experimental procedures in El-Kaser El-Eini animal house, Faculty of Medicine, Cairo University. The surgical procedure was done by a specialized veterinarian in the animal house, Faculty of Medicine, Cairo University. Each animal was sedated with an intramuscular injection of $1 \mathrm{mg} / \mathrm{Kg}$ xylazine. General anaesthesia was introduced using sodium pentobarbital.

Permanent mandibular molars and premolars were selected as the most suitable teeth in 6 male mongrel dogs. All teeth were scaled and polished with a rubber cup, on the day of the operative procedure. Quadrants of teeth (3 premolars and 2 molars) were isolated using the rubber dam, and saliva was controlled through highspeed evacuation. Occlusal pulp exposures were performed using a round carbide bur $(0.8 \mathrm{~mm}$ in diameter), at high speed and under water cooling. The cavities were then washed with sterile saline and dried with cotton pellets. Light pressure was applied so that haemorrhage could be controlled. The pulp exposures in each dog was randomly capped in pairs according to the manufacturer instructions with MTA (Dentsply Tulsa Dental, Tulsa, OK, USA), BM-MSCs and BA (Innovative Bio-Ceramix, Vancouver, British Columbia, Canada), where MTA was used as the control and placed in a single pair of teeth. The cavities were restored with zinc oxideeugenol and amalgam. Experimental procedures in the six dogs were conducted in the same way. The pulpal tissue responses to the tested materials were assessed postoperatively after sacrificing the dogs by injecting an overdose of pentobarbital sodium after 30 days. Teeth were extracted and fixed in $10 \%$ neutral buffered formalin solution. After decalcification in formic acid-sodium citrate solution, the teeth were embedded in paraffin. Serial sections $(6-\mu \mathrm{m}$ thick) were stained with hematoxylin and eosin. Specimens were divided into three groups according to the capping material used into MTA (group I), BA (group II) and BMMSCs (group III). During the analysis of the results of this study, details were considered regarding the hard tissue bridge (continuity, morphological aspects, and thickness), and inflammatory reaction.

\section{Isolation and Culture of BM-MSCs:}

Bone marrow (20 ml/sample) was aspirated from the iliac crest of mongrel dogs under general anaesthesia using a Rothensal bone marrow biopsy needle. Bone marrow samples were transferred for further processing and culture in tissue culture unit at medical biochemistry and molecular biology unit in the faculty of medicine Cairo University. The BM-MSCs were cultured in minimal essential medium (MEM) supplemented with 10\% fetal bovine 
serum (FBS) and $0.5 \%$ penicillin, streptomycin at $37^{\circ} \mathrm{C}$ in $5 \% \mathrm{CO}_{2}$ in the air.

\section{RNA Extraction}

Paraffin was dissolved from tissues in all studied groups. Purified tissue was lysed, and total RNA was isolated with RNAeasy Mini Kit (Qiagen) and further analyzed for quantity and quality with Beckman dual spectrophotometer (USA).

\section{Real-Time PCR (qRT-PCR) for Quantitative Ex- pression of $\beta$-catenin:}

The mRNA expression level was quantified by qRT-PCR (Real time PCR) blindly. The total RNA $(1 \mu \mathrm{g})$ from each sample was used for cDNA synthesis by reverse transcription using high capacity cDNA Reverse Transcriptase kit (Applied Biosystem, USA). The cDNA was subsequently amplified with the Syber Green I PCR Master Kit (Fermentas) in a 48-well plate using the Step One instrument (Applied Biosystem, USA) as follows: 10 minutes at $95^{\circ} \mathrm{C}$ for enzyme activation followed by 40 cycles of 15 seconds at $95^{\circ} \mathrm{C}, 20$ seconds at $60^{\circ} \mathrm{C}$ and 30 second at $72^{\circ} \mathrm{C}$ for the amplification step. Changes in the expression of each target gene were normalized relative to the mean critical threshold (CT) values of GAPDH housekeeping gene by the $\Delta \Delta \mathrm{Ct}$ method. Both primers $(1 \mu \mathrm{M})$ specific for each target gene were used. Primers sequence and annealing temperature specific for each gene are demonstrated in table (1) and figure (1).

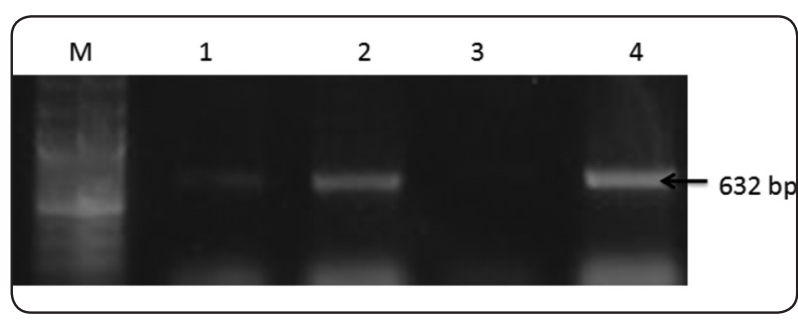

Fig. (1) Agarose gel electrophoresis showed PCR product (632 bp) of $\beta$-catenin gene expression in all studied groups. Lane M: DNA marker. Lane 1: PCR product of F, Lane 2: PCR product of E, Lane 3: negative control and Lane 4: PCR product of $\mathrm{D}$

\section{Statistical analysis}

Data presented as means and standard deviation (SD) values. Data explored for normality using Kolmogorov Smirnov test. Data showed parametric distribution so; One Way ANOVA was used to compare between the tested materials followed by Tukey's Post Hoc test for pairwise comparison. The significance level was set at $\mathrm{P} \leq 0.001$. Statistical analysis was performed with IBM ${ }^{\circledR}$ SPSS ${ }^{\circledR}$ (SPSS Inc., IBM Corporation, NY, USA) Statistics Version 24 for Windows.

\section{RESULTS}

\section{Histological results}

Sections of the MTA specimens (group I) showed, hard tissue bridge beneath the pulp exposure site with regular contour (Fig. 2). In BA specimens (group II) complete calcified bridge

TABLE (1) Primers sequence and annealing temperature specific for each gene

\begin{tabular}{|c|c|c|}
\hline Target gene & Primer sequence: 5 $^{`}$ - ${ }^{`}$ & Gene bank accession number \\
\hline B-catenin & $\begin{array}{c}\text { Forward: GGACCTCAGCAAGATCATGGC } \\
\text { Reverse: CCACGATCTTACGGGTAGTTG }\end{array}$ & XM008772775.1 \\
\hline GAPDH & Forward :CACCCTGTTGCTGTAGCCATATTC & XR598347.1 \\
& Reverse: GACATCAAGAAGGTGGTGAAGCAG & \\
\hline
\end{tabular}


at the exposure site was observed. In few cases, porous, non- homogenous dentin bridges were noticed (Fig. 3). In of BM-MSCs specimens (group III) the exposure site was completely closed by a dentin bridge with irregular contour. (Fig. 4). In all groups, few inflammatory cells were observed with no evidence of necrosis.

\section{$\beta$-catenin expression levels}

BM-MSCs $(5.63 \pm 0.09)$ showed the highest $\beta$-catenin expression level followed by BA $(3.42 \pm 0.17)$ followed by the MTA $(2.34 \pm 0.16)$ at $\mathrm{p} \leq 0.001$. (Table 2, Fig 5).

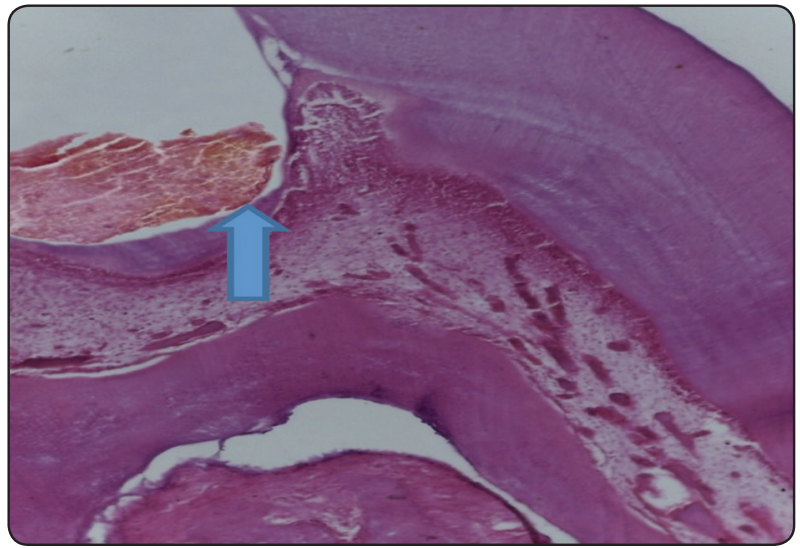

Fig. (2) A Photomicrograph of a dog's tooth of group I (MTA) at 30 days showed a homogeneous dentin bridge (blue arrow) (H \& EX100).

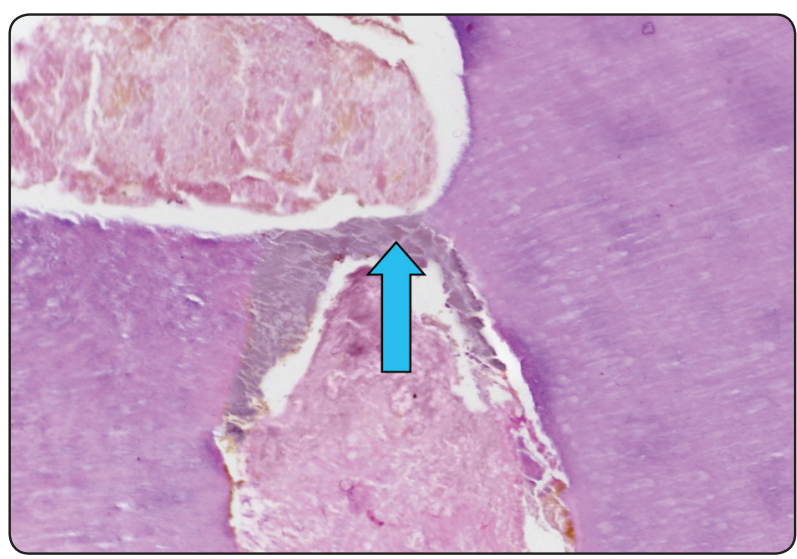

Fig. (3) A Photomicrograph ofa dog's tooth of group II (BA) at 30 days showed non- homogeneous irregular dentin bridge (blue arrow) (H \& EX100)

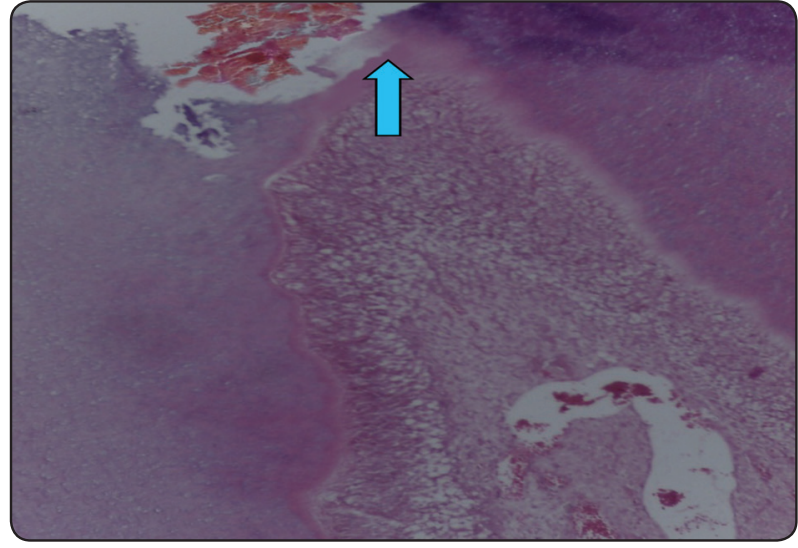

Fig. (4) A photomicrograph of a dog's tooth of group III (BMMSCs) at 30 days showed continuous dentin bridge under the capping material (blue arrow) (H\&EX100).

TABLE (2) Mean, standard deviation (SD)for the $\beta$-catenin expression levels in all tested capping materials

\begin{tabular}{|c|c|c|c|c|c|c|c|}
\hline & \multicolumn{2}{|c|}{ BM-MSCs } & \multicolumn{2}{|c|}{ BA } & \multicolumn{2}{c|}{ MTA } & \multirow{2}{*}{ p-value } \\
\cline { 2 - 7 } & Mean & SD & Mean & SD & Mean & SD & \\
\hline $\begin{array}{c}\beta \text {-catenin } \\
\text { protein }\end{array}$ & $5.63^{\mathrm{a}}$ & 0.09 & $3.42^{\mathrm{b}}$ & 0.17 & $2.34^{\mathrm{c}}$ & 0.16 & $\leq 0.001^{*}$ \\
\hline
\end{tabular}

Means with the same letter within row are statistically insignificant at $p>0.05$

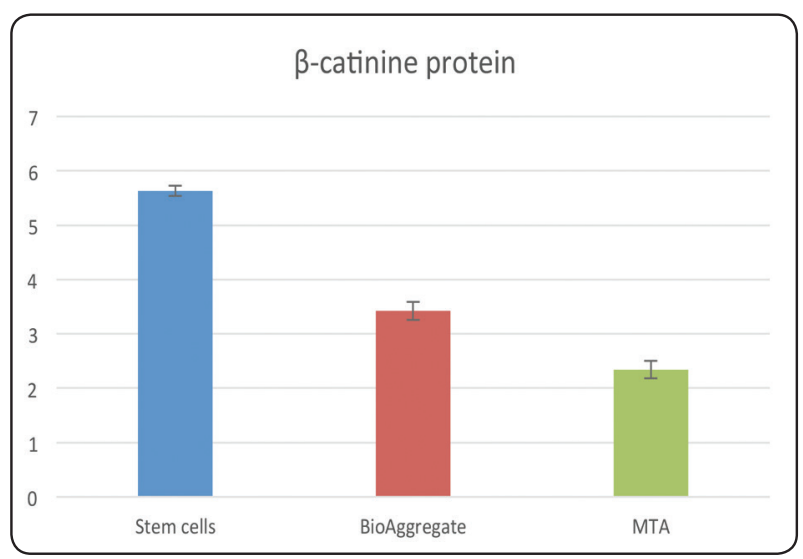

Fig. (5) Bar chart showing the $\beta$-catenin expression levels in all tested capping materials 


\section{DISCUSSION}

Dental pulp vitality is important for tooth survival. Direct pulp capping is the sealing of mechanical pulp exposure with biomaterials placed directly on exposed pulp to induce reparative dentin formation ${ }^{(33)}$.

Histological results in the present study revealed formation of a dentin bridge in all groups. Regarding MTA group, our finding was in accordance to Nair et al. 2008 who demonstrated uniform dentin bridge formation with MTA and added that the formation of hard tissue barrier is multifactorial, involving its sealing ability ,biocompatibility and production of an alkaline pulpal environment ${ }^{(14)}$. Also, Aeinehchi et al. 2003 suggested that pulp capping with MTA induced cytological and functional changes in the pulp cells leading to formation of reparative dentin ${ }^{(34)}$.

Concerning BA group, the current histological result (formation of complete dentin bridge at the exposure site) was in agreement with Jia et al. 2016, who demonstrated, a dense calcified area at the pulp exposure interface in Bioaggregate specimens after 4 weeks ${ }^{(35)}$. Zhang et al. 2013, reported that Bioaggregate exerts a great odontogenic potential as it induced mineralization and odontoblastic differentiation-assiocated gene expression in human dental pulp cells ${ }^{(36)}$.

In the current study, most specimens of group III (BM-MSCs) showed considerable dentin bridge at the exposure site. This result was in agreement with Sugiyama et al. 2011, who reported that pulp stem cells induce angiogenesis in rat cerebral ischemia by releasing neurotrophic factors such as vascular endothelial growth factor (VEGF) ${ }^{(37)}$. Angiogenesis is the process of new blood vessel formation from preexisting vasculature. Conservative pulp treatments such as direct pulp capping trigger wound healing events that are orchestrated by an exquisitely regulated angiogenic response ${ }^{(38)}$. Also in accordance with our result, Tziafas et al. 2000 reported that damaged odontoblasts can also be replaced by newly generated populations of odontoblasts derived from stem cells from pulp. Following physiological stimulation or injury, such as caries and operative procedures, stem cells in pulp may be mobilized to proliferate and differentiate into odontoblasts by morphogenes released from the surrounding dentin matrix ${ }^{(39)}$. $\beta$-catenin is a key player in tooth development, especially in odontoblast differentiation. It regulates Runx2, transcriptional factor and master regulator of odontoblast and osteoblast differentiation. Moreover, Runx2 activates the transcription of dentin sialophosphoprotein gene which encodes two specific dentin proteins; dentin sialoprotein and dentin phosphoprotein. $\beta$-catenin levels have been shown to be up-regulated in the odontoblast during reparative dentinogenesis ${ }^{(32)}$. The results of the current study revealed the greatest level of $\beta$ catenin expression in group III, followed by group II, while the lowest level was in group I. Statistical analysis of the data also revealed a high statistically significant difference among the studied groups. From all the previously mentioned data it could be concluded that dentinogenesis was most active in group III (BM-MSC).

\section{CONCLUSIONS}

Although the dentinogenesis process was most active in the BM-MSCs group the decision to incorporate stem cell-based therapies into routine clinical dental practice requires careful analysis of the risks and benefits associated with the procedure.

\section{REFERENCES}

1. Schroder U. Effects of calcium hydroxide-containing pulpcapping agents on pulp cell migration, proliferation, and differentiation. J Dent Res 1985; 64: 541-8.

2. Ricketts D. Management of the deep carious lesion and the vital pulp dentine complex. Br Dent J. 2001; 191: 606-10.

3. Stanley HR. Pulp capping: conserving the dental pulp--can it be done? Is it worth it? Oral Surg Oral Med Oral Pathol. 1989; 68: 628-39. 
4. Demarco FF, Rosa MS, Tarquínio SB, Piva E. Influence of the restoration quality on the success of pulpotomy treatment: a preliminary retrospective study. J Appl Oral Sci. 2005; 13: 72-7.

5. Holland R, Filho JA, de Souza V, Nery MJ, Bernabé PF, Junior ED. Mineral trioxide aggregate repair of lateral root perforations. J Endod 2001; 27: 281-4.

6. Faraco IM Jr, Holland R. Response of the pulp of dogs to capping with mineral trioxide aggregate or a calcium hydroxide cement. Dent Traumatol 2001; 17: 163-6.

7. Dammaschke T, Wolff P, Sagheri D, Stratmann U, Schäfer E. Mineral trioxide aggregate for direct pulp capping: a histologic comparison with calcium hydroxide in rat molars. Quintessence Int 2010; 41: e20-30.

8. Parirokh M, Torabinejad M. Mineral trioxide aggregate: A comprehensive literature review-part III: clinical applications, drawbacks, and mechanism of action. J Endod 2010; 36: 400-413.

9. Sawicki L1, Pameijer CH, Emerich K, AdamowiczKlepalska B. Histological evaluation of mineral trioxide aggregate and calcium hydroxide in direct pulp capping of human immature permanent teeth. Am J Dent. 2008;21:262-6.

10. Ford TR, Torabinejad M, McKendry DJ, Hong CU, Kariyawasam SP. Use of mineral trioxide aggregate for repair of furcal perforations. Oral Surg Oral Med Oral Pathol Oral Radiol Endod 1995; 79: 756-63.

11. Ford TR, Torabinejad M, Abedi HR, Bakland LK, Kariyawasam SP. Using mineral trioxide aggregate as a pulpcapping material. J Am Dent Assoc 1996; 127: 1491-4.

12. Paranjpe A, Zhang H, Johnson JD. Effects of mineral trioxide aggregate on human dental pulp cells after pulp-capping procedures. J Endod 2010;36 :1042-7.

13. Gomes AC, Filho JE, de Oliveira SH. MTA-induced neutrophil recruitment: a mechanism dependent on IL-1beta, MIP-2, and LTB4. Oral Surg Oral Med Oral Pathol Oral Radiol Endod 2008;106:450-6.

14. Nair PN, Duncan HF, Pitt Ford TR, Luder HU. Histological, ultrastructural and quantitative investigations on the response of healthy human pulps to experimental capping with mineral trioxide aggregate: a randomized controlled trial. Int Endod J 2008;41:128-50.

15. Paranjpe A, Zhang H, Johnson JD. Effects of mineral trioxide aggregate on human dental pulp cells after pulp-capping procedures. J Endod 2010;36:1042-7.
16. Paranjpe A, Cacalano NA, Hume WR, Jewett A. N-acetylcysteine protects dental pulp stromal cells from HEMAinduced apoptosis by inducing differentiation of the cells. Free Radic Biol Med 2007;43:1394-408.

17. Barkhordar RA, Ghani QP, Russell TR, Hussain MZ. Interleukin-1beta activity and collagen synthesis in human dental pulp fibroblasts. J Endod 2002;28:157-9.

18. Cavalcanti BN, Rode Sde M, França CM, Marques MM. Pulp capping materials exert an effect on the secretion of IL-1beta and IL-8 by migrating human neutrophils. Braz Oral Res 2011;25:13-8.

19. Ferreira DC, Brito DG, Cavalcanti BN. Cytokine production from human primary teeth pulp fibroblasts stimulated by different pulpotomy agents. J Dent Child (Chic) 2009;76:194-8.

20. Barbosa Silva MJ, Vieira LQ, Sobrinho AP. The effects of mineral trioxide aggregates on cytokine production by mouse pulp tissue. Oral Surg Oral Med Oral Pathol Oral Radiol Endod 2008;105:e70-6.

21. Rezende TM, Vieira LQ, Sobrinho AP, Oliveira RR, Taubman MA, Kawai T. The influence of mineral trioxide aggregate on adaptive immune responses to endodontic pathogens in mice. J Endod 2008;34:1066-71.

22. Zhang H, Pappen FG, Haapasalo M. Dentin enhances the antibacterial effect of mineral trioxide aggregate and bioaggregate. J Endod 2009;35:221-4.

23. Damas BA, Wheater MA, Bringas JS, Hoen MM. Cytotoxicity comparison of mineral trioxide aggregates and EndoSequence bioceramic root repair materials.J Endod 2011;37:372-5.

24. Alanezi AZ, Jiang J, Safavi KE, Spangberg LS, Zhu Q. Cytotoxicity evaluation of endosequence root repair material. Oral Surg Oral Med Oral Pathol Oral Radiol Endod 2010; 109:e122-5.

25. De-Deus G, Canabarro A, Alves G, Linhares A, Senne MI, Granjeiro JM. Optimal cytocompatibility of a bioceramic nanoparticulate cement in primary human mesenchymal cells. J Endod 2009;35:1387-90.

26. Yan P, Yuan Z, Jiang H, Peng B, Bian Z. Effect of bioaggregate on differentiation of human periodontal ligament fibroblasts. Int Endod J 2010; 43: 1116-21.

27. Yuan Z, Peng B, Jiang H, Bian Z, Yan P. Effect of bioaggregate on mineral-associated gene expression in osteoblast cells. J Endod 2010; 36: 1145-8. 
28. Ciasca M, Aminoshariae A, Jin G, Montagnese T, Mickel A. A comparison of the cytotoxicity and proinflammatory cytokine production of EndoSequence root repair material and ProRoot mineral trioxide aggregate in human osteoblast cell culture using reverse-transcriptase polymerase chain reaction. J Endod 2012; 38 :486-9.

29. Van der Kooy D, Weiss S. Why stem cells. Science 2000; 287: 1439-41.

30. Fortier LA. Stem cells: classifications, controversies, and clinical applications. Vet Surg 2005;34:415-23.

31. PetersOA.Translational opportunities in stem cellbased endodontic therapy: where are we and what are we missing? J Endod. 2014; 40: S82-5.

32. Han N, Zheng Y, Li R, Li X, Zhou M, Niu Y, Zhang Q. $\beta$-catenin enhances odontoblastic differentiation of dental pulp cells through activation of Runx2. PLoS One. 2014;10;9(2):e88890.

33. Tziafas D. The future role of a molecular approach to pulpdentinal regeneration. Caries Res. 2004; 38: 314-20.

34. Aeinehchi M, Eslami B, Ghanbariha M, Saffar AS. Mineral trioxide aggregate (MTA) and calcium hydroxide as pulp-capping agents in human teeth: a preliminary report. Int Endod J 2003; 36: 225-31.

35. Jia Kim, Young-SangSong, Kyung-San Min, Sun-Hun Kim, Jeong-Tae Koh, Bin-Na Lee1, Hoon-Sang Chang, In-Nam Hwang, Won-Mann Oh, Yun-Chan Hwang. Evaluation of reparative dentin formation of ProRoot MTA, Biodentine and BioAggregate using micro-CT and immunohistochemistry. RDE J. 2016, 41: 29-36.

36. Zhang S, Yang X, Fan M. BioAggregate and iRoot BP Plus optimize the proliferation and mineralization ability of human dental pulp cells. Int Endod J 2013; 46: 923-29.

37. Sugiyama, M, Iohara, K, Wakita, H, Hattori, H, Jueda, M. \& Nakashima, M. Dental pulp-derived CD31/ CD146 side population stem/progenitor cells enhance recovery of focal celebral ischemia in rats. Tissue Eng part A 2011; 17: 1303-11.

38. Nakashima, M.\& Akamine, A. The application of tissue engineering to regeneration of pulp and dentin in endodontics. J Endod 2005; 31: 711-18.

39. Tziafas D, Smith AJ, Lesot H. Designing new treatment strategies in vital pulp therapy. J Dent 2000; 28:77-92. 